\title{
A TWO-GRID TIME-DEPENDENT FORMALISM FOR THE MAXWELL EQUATION
}

\author{
DANIEL NEUHAUSER \\ Department of Chemistry and Biochemistry, UCLA, Los Angeles, CA 90095-1569, USA \\ ROI BAER \\ Institute of Chemistry, Lisa Meitner Center, Hebrew University of Jerusalem, \\ Jerusalem 91904, Israel
}

\begin{abstract}
We develop a formalism for efficient iterative solutions of scattering problems involving the Maxwell equations. The methods, borrowed from recent advancements in chemical reaction dynamics, represent the scattering wavefunctions on two grids; one used for the initial wave and is one-dimensional; the other is a small three-dimensional grid padded with absorbing-potentials on which the scattered function is represented. The formalism is automatically suitable for scattering studies of transmission, reflection and scattering components of a wave. The simulations can be done with time-dependent wavepackets or direct iterative solution for the Green's function, but the results are rigorous time-independent (frequencydependent) scattering amplitudes. Model time-dependent simulations involving up to a $100 \times 100 \times 100$ grid for the inner wavefunction were numerically done on a simple PC.
\end{abstract}

Keywords: Maxwell equation; photonic crystals; time-dependent methods.

\section{Introduction}

The study of photonic band-gap materials is increasing in importance. ${ }^{1-6}$ Together with the experimental studies, it is important to develop simulation tools for studying specific properties. ${ }^{7-9}$ The simplest properties are band-gaps of periodic systems. However, there are other interesting problems which are more difficult to simulate. These include specifically the scattering and transmission probabilities from a perturbation in the material ${ }^{10-12}$ as well as the scattering from a timedependent perturbation.

The problem is analogous to large-scale scattering problems of the Schrödinger equation. About ten years ago, there was a flurry of interest in the development of chemical reaction dynamics methods (for a review see Neuhauser et al. ${ }^{13}$ ) with specific development of several tools for the study of very large scale problems including systems of 6 or more dimension, which is what happens in four-body scattering ${ }^{14-17}$ or surface scattering. ${ }^{18}$ These tools included, among others, the development of iterative techniques which are based on wave-packets ${ }^{19-23}$ and so are able to give immediate results at many energies; the use of absorbing (negative imaginary) potentials for reducing the grid size ${ }^{24}$ and the use of projection operator formalisms to turn the scattering problems into bound-state-like problem where the grids need to cover essentially only the strong-interaction region. ${ }^{25,26}$

In this work we outline the application of similar techniques for the Maxwell equation. The idea of using wave-packet techniques for solving the Maxwell equation is not new. ${ }^{27,28}$ But this work is distinguished by using the complete suite of development from time-dependent reactive scattering techniques; an in particular the use of a two-grid approach. 
The two-grid approaches aim to solve an inherent problem in scattering techniques. On the one hand, the initial wavefunction in scattering lies away from the strong interaction region. On the other hand, the physics of the scattering occurs only in a limited region so that it should be feasible to restrict the numerical modeling into a small strong interaction region. The solution is to divide the wavefunction into two parts: one contains the initial wavefunction and is one-dimensional; the other is multidimensional but covers only a small strong interaction region, padded with absorbing potentials. There are two possibilities for dissecting the wavefunction to two parts in chemical dynamics: one in which the wavefunction on the 1-D grid is obtained by a projection-operator, ${ }^{23}$ and one in which it is a solution of a simpler zeroorder Hamiltonian; ${ }^{26}$ only the second approach would be relevant here, as mentioned below.

The two-grid formalism solves a difficulty in the simulation of electromagnetic waves with wave-packet techniques. The formal initial state is typically a waverfront extended over an infinitely large region (perpendicular to the reaction direction). However, if a single grid is used, it is impossible to describe the initial single infinitely extended state in $x-y$. The two-grid formalism solves this problem while retaining the homogeneity of the propagation equations; the homogeneity is important since it allows the use of time dependent techniques for extracting many energy values from one wave-packet. Incidentally, the situation is similar for the scattering of associating molecules in chemical dynamics, where two-grid formalisms could also be very useful.

This paper lays down the basic theory and techniques, and uses a simple system to demonstrate the method. The theory is presented in stages; first, the transformation of the Maxwell equation to the Schrödinger equation is presented (analogous treatments are prevalent). The most interesting aspect is the form of the flux operator, which does not involve a derivative directly. The formulation in time-dependent language is presented later, followed by the development of the two-grid formalism. Model simulations follow, and the paper finishes with a discussion and conclusions. An appendix maps several other chemical-scattering formulations in the Maxwell problem.

\section{Methodology}

Maxwell's equations are discussed here for nonmagnetic media $(\mu=1)$ and without currents and take the following simple form:

$$
\begin{gathered}
\frac{\partial \varepsilon \mathbf{E}}{\partial t}=\nabla \times \mathbf{B} \\
\frac{\partial \mathbf{B}}{\partial t}=-\nabla \times \mathbf{E} .
\end{gathered}
$$

(The cases of magnetic media and currents can also be included, as will be shown in future work). We work in a system of units where $c=1$ and the vacuum permittivity is 1 . We will use bold-face letters for 3dimensional vectors. The vector $\mathbf{A}$ with a caret will denote the corresponding unit vector: $\hat{\mathbf{A}}=\mathbf{A} /|\mathbf{A}|$.

Equations (1) and (2) are easily converted into Hamiltonian-like equations. We define a super-vector:

$$
\psi \equiv\left(\begin{array}{c}
\sqrt{\varepsilon \mathbf{E}} \\
\mathbf{B}
\end{array}\right) .
$$

which satisfying a Schrödinger-like equation:

$$
i \frac{\partial \psi}{\partial t}=H \psi
$$

where

$$
H=\left(\begin{array}{cc}
0 & \frac{i}{\sqrt{\varepsilon}} \nabla \times \\
-\nabla \times \frac{i}{\sqrt{\varepsilon}} & 0
\end{array}\right)
$$

is the Schrödinger-like "Hamiltonian" giving rise to the Maxwell equation (this operator should not be confused, of course, with the Field-Theoretical Hamiltonian of the electromagnetic field; the only reason we label $H$ as an Hamiltonian is due to its formal similarity to Schrödinger equation). $H$ is imaginary, but the propagation can be completely real (details would be provided in an upcoming paper).

$H$ is manifestly Hermitian (except for the added absorbing potential and the two-grid formalism presented below). All the known scattering methodologies can be applied for this Hamiltonian, with particular care to the initial and final states. 


\subsection{Zero-order Hamiltonian}

The first step is to define a zero-order Hamiltonian, $H_{0}$ as:

$$
H_{0}=\left(\begin{array}{cc}
0 & \frac{i}{\sqrt{\varepsilon_{b}}} \nabla \times \\
-\nabla \times \frac{i}{\sqrt{\varepsilon_{b}}} & 0
\end{array}\right),
$$

where $\varepsilon_{b}$ is the permittivity of the background. This background could be one of several choices of varying complexity.

In the simplest case, the background would be of constant permittivity, as happens when the scattering is incident from a homogeneous isotropic crystal or from air. Alternately, it could be periodic along a single axis, either the dimension of propagation defined as $z$ [so $\varepsilon_{b}=\varepsilon(z)$ ], or another direction, and constant in the perpendicular directions. It could be periodic in three dimensions, one of which is the dimension of propagation. In the most general case, it could be periodic in three dimensions of arbitrary orientations.

The formalism is appropriate, with simple modification, for all cases above. For clarity, it is presented in this paper for the first case (constant background). Future works present the extension to periodic backgrounds.

\subsection{Scattering states}

The next step is to define a scattering state. The timeindependent asymptotic purely outgoing scattering states of $H_{0}$ are denoted as $\psi_{0}(\mathbf{r} ; \mathbf{k}, \hat{\mathbf{p}})$, where we defined the $3-\mathrm{D}$ position vector $\mathbf{r}$, the propagation axis and the polarization vector. For constant background, $\psi_{0}(\mathbf{r} ; \mathbf{k}, \hat{\mathbf{p}})$ is a plane wave:

$$
\psi_{0}(\mathbf{r} ; \mathbf{k}, \hat{\mathbf{p}})=\frac{1}{\sqrt{16 \pi^{3}}} \exp (i \mathbf{k} \cdot \mathbf{r}) f_{\hat{p}},
$$

where

$$
f_{\hat{p}}=\left(\begin{array}{c}
\hat{\mathbf{p}} \\
\hat{\mathbf{k}} \times \mathbf{p}
\end{array}\right) .
$$

For this case, the state $\psi_{0}(\mathbf{r} ; \mathbf{k}, \hat{\mathbf{p}})$ is an eigenstate of $H_{0}$ :

$$
H_{0} \psi_{0}=\omega \psi_{0},
$$

with the frequency, or in analogy to Schrödinger equation, the "energy" (we will use the terms energy and frequency interchangeably) independent of the polarization:

$$
\omega(\mathbf{k})=\frac{|\mathbf{k}|}{\sqrt{\varepsilon_{b}}} .
$$

The pre-factors in Eq. (7) are such that the following normalization holds:

$$
\left\langle\psi_{0}(\mathbf{k}, \hat{\mathbf{p}}) \mid \psi_{0}\left(\mathbf{k}^{\prime}, \hat{\mathbf{p}}^{\prime}\right)\right\rangle=\delta\left(\mathbf{k}-\mathbf{k}^{\prime}\right) \hat{\mathbf{p}} \cdot \hat{\mathbf{p}}^{\prime} .
$$

Consider now a scattering state of the total Hamiltonian $H$, which reduces to $H_{0}$ for $|\mathbf{r}| \rightarrow \infty$. Denote this state as $\psi_{\text {scatt }}\left(\mathbf{r} ; \mathbf{k}_{i}, \hat{\mathbf{p}}_{i}\right)$. It corresponds to an incoming wave $\psi_{\text {in }}(\mathbf{r})$ of a given wave vector $\mathbf{k}_{i}$ and polarization $\hat{\mathbf{p}}_{i}$ where:

$$
\psi_{\text {in }}(\mathbf{r})=\psi_{0}\left(\mathbf{r} ; \mathbf{k}_{i}, \hat{\mathbf{p}}_{i}\right)
$$

The scattering state is also an eigenstate of $H$ with the same energy as the incoming wave:

$$
H \psi_{\text {scatt }}=\omega \psi_{\text {scatt }} .
$$

We decompose the scattering state to a sum of the incoming and outgoing components:

$$
\begin{aligned}
\psi_{\text {scatt }}\left(\mathbf{r} ; \mathbf{k}_{i}, \hat{\mathbf{p}}_{i}\right) & =\psi_{\text {in }}+\psi_{\text {out }} \\
& \equiv \psi_{0}\left(\mathbf{r} ; \mathbf{k}_{i}, \hat{\mathbf{p}}_{i}\right)+\psi_{\text {out }}\left(\mathbf{r} ; \mathbf{k}_{i}, \hat{\mathbf{p}}_{i}\right) .
\end{aligned}
$$

Asymptotically, i.e. far from an interaction region, the outgoing part is a superposition of plane waves with the same energy $\omega$. The amplitudes of each planewave component is given by the T-matrix, defined by:

$$
\begin{aligned}
& \psi_{\text {out }}\left(|\mathbf{r}| \rightarrow \infty ; \mathbf{k}_{i}, \hat{\mathbf{p}}_{i}\right) \\
& \quad=2 \pi \sum_{p} \int t\left(\mathbf{k}, \mathbf{k}_{i}, \hat{\mathbf{p}}, \hat{\mathbf{p}}_{i}\right) \delta(\omega(\mathbf{k})-\omega) \psi_{0}(\mathbf{r} ; \mathbf{k}, \hat{\mathbf{p}}) d^{3} \mathbf{k} .
\end{aligned}
$$

While we chose an example where $H_{0}$ is of constant "potential" $\varepsilon_{b}$, other choices can also be made. In particular problems, $H_{0}$ can be chosen with a reflective potential, for example. In this case, the "incoming" wave will not be a pure plane wave and will include the reflective part as well.

\subsection{The flux operator}

The flux through a closed surface $S$ is defined in quantum mechanics: ${ }^{29}$

$$
F=i[H, \theta]
$$


Here $\theta=\theta(\mathbf{r})$ is a function defining the surface by assigning the value 1 to any point with the surface and 0 to any point outside it. The surface can be infinite (for example, an infinite plane) which divides space into two regions: one is considered "inside", and $\theta$ has the value 1 on it and the other "outside" with $\theta=0$ on it. ${ }^{a} F$ is the flux operator in the boundary surface. Carrying the notion of flux to the present study, it is straightforward to prove, using Eq. (5) in (16) that:

$$
F=\left(\begin{array}{cc}
0 & -\frac{\nabla \theta}{\sqrt{\varepsilon}} \times \\
\frac{\nabla \theta}{\sqrt{\varepsilon}} \times & 0
\end{array}\right) .
$$

For our purpose here, $S$ is taken as a plain surface, defined by the equation $s(\mathbf{r}) \equiv \hat{\mathbf{a}} \cdot \mathbf{r}=s_{0}$, where $\hat{\mathbf{a}}$ is a unit vector perpendicular to the surface and $s_{0}$ is a large (nonzero) number, so that the surface is far from the interaction region. Then:

$$
\theta(\mathbf{r})=h\left(s_{0}-s(\mathbf{r})\right),
$$

where $h(x)=1$ when $x>0$ and 0 otherwise. Since at large $\mathbf{r} \varepsilon$ is constant, this leads, in an obvious notation, to:

$$
F=\left(\begin{array}{cc}
0 & -\frac{\delta}{\sqrt{\varepsilon}} \hat{\mathbf{a}} \times \\
\frac{\delta}{\sqrt{\varepsilon}} \hat{\mathbf{a}} \times & 0
\end{array}\right) .
$$

From this expression, it is straightforward to show that, in analogy to the usual Schrödinger equation, the following relation holds:

$$
\begin{aligned}
&\left\langle\psi_{0}\left(\mathbf{k}^{\prime}, \hat{\mathbf{p}}^{\prime}\right)|F| \psi_{0}(\mathbf{k}, \hat{\mathbf{p}})\right\rangle_{\omega(\mathbf{k})=\omega\left(\mathbf{k}^{\prime}\right)} \\
& \quad=\frac{\hat{\mathbf{k}} \cdot \hat{\mathbf{a}}}{2 \pi} \delta\left(\mathbf{k}_{\perp}-\mathbf{k}_{\perp}^{\prime}\right) \delta_{\operatorname{sign}(\mathbf{k} \cdot \mathbf{a}), \operatorname{sign}\left(\mathbf{k}^{\prime} \cdot \mathbf{a}\right) \hat{\mathbf{p}} \cdot \hat{\mathbf{p}}^{\prime}}
\end{aligned}
$$

Let us discuss the terms in Eq. (20). Since we are at the asymptotes, there is full $\mathbf{k}$ vector conservation of a plane wave crossing the surface $S$. Since we already consider the same energy, it remains only to conserve the perpendicular components of $\mathbf{k}$ and the sign of $\hat{\mathbf{k}} \cdot \hat{\mathbf{a}}$. The factor $\hat{\mathbf{a}} \cdot \hat{\mathbf{k}} / 2 \pi$ takes into account the direction of the wave with respect to the surface.

\subsection{The transition amplitude}

Combining expressions (15) and (20), we get the transition amplitude in any direction $\mathbf{k}$ by considering the quantity $\left\langle\psi_{0}(\mathbf{k}, \hat{\mathbf{p}})|F| \psi_{\text {out }}\left(\mathbf{k}_{i}, \hat{\mathbf{p}}\right)\right\rangle$ for two asymptotic surfaces defined by $s(\mathbf{r})=\hat{\mathbf{k}}_{i} \cdot \mathbf{r}=s_{0}$ : one surface is with $s(\mathbf{r})>0$ and the other $s(\mathbf{r})<0$. For the positive case $\left(s_{0} \gg k^{-1}\right)$ :

$$
\begin{aligned}
& \left\langle\psi_{0}(\mathbf{k}, \hat{\mathbf{p}})|F| \psi_{\text {scatt }}\right\rangle_{s_{0} \gg k^{-1}} \\
& \quad=\frac{1}{2 \pi} \delta\left(\mathbf{k}_{\perp}-\mathbf{k}_{i \perp}\right) \delta_{\operatorname{sign}\left(\hat{\mathbf{k}} \hat{\mathbf{k}}_{i}\right)} \hat{\mathbf{p}} \cdot \hat{\mathbf{p}}_{i}+t\left(\mathbf{k}, \mathbf{k}_{i}, \hat{\mathbf{p}}, \hat{\mathbf{p}}_{i}\right) \frac{d k_{\|}}{d \omega} .
\end{aligned}
$$

The first term is due to the incoming wave and the second arises from the outgoing:

$$
\begin{aligned}
\left\langle\psi_{0}(\mathbf{k}, \hat{\mathbf{p}})|F| \psi_{\text {out }}\right\rangle_{s_{0} \gg k^{-1}} & =2 \pi \int t\left(\mathbf{k}^{\prime}, \mathbf{k}_{i}, \hat{\mathbf{p}}^{\prime}, \hat{\mathbf{p}}_{i}\right) \frac{\delta\left(\mathbf{k}_{\perp}-\mathbf{k}_{\perp}^{\prime}\right)}{2 \pi} \delta(\omega(\mathbf{k})-\omega) d^{3} \mathbf{k}^{\prime} \\
& =\int t\left(\mathbf{k}^{\prime}, \mathbf{k}_{i}, \hat{\mathbf{p}}^{\prime}, \hat{\mathbf{p}}_{i}\right) \delta\left(\mathbf{k}_{\perp}-\mathbf{k}_{\perp}^{\prime}\right) \delta\left(k_{\|}-k_{\|}^{\prime}\right) \frac{\partial k_{\|}}{\partial \omega} d^{3} \mathbf{k}^{\prime} \\
& =t\left(\mathbf{k}, \mathbf{k}_{i}, \hat{\mathbf{p}}, \hat{\mathbf{p}}_{i}\right) \frac{d k}{d \omega}
\end{aligned}
$$

Here we used the fact that the surface is defined along $\hat{\mathbf{k}}$, so that $k_{\|}=k$. Note also that we explicitly used the fact that the flux is evaluated for large positive $s_{0}$. Upon repeating the calculation with $\left|s_{0}\right|$ large but $s_{0}$ negative - i.e. a surface which, relative to $\mathbf{k}$, is way in the back, the $T$-matrix term drops and:

$$
\begin{aligned}
& \left\langle\psi_{0}(\mathbf{k}, \hat{\mathbf{p}})|F| \psi_{\text {scatt }}\right\rangle_{s_{0} \ll-k^{-1}} \\
& \quad=\frac{1}{2 \pi} \delta\left(\mathbf{k}_{\perp}-\mathbf{k}_{i \perp}\right) \delta_{\operatorname{sign}\left(\hat{\mathbf{k}} \cdot \hat{\mathbf{k}}_{i}\right)} \hat{\mathbf{p}} \cdot \hat{\mathbf{p}}_{i} .
\end{aligned}
$$

\footnotetext{
${ }^{\mathrm{a}}$ It is not strictly necessary that the transition between the 1 and 0 be abrupt — the formalism is equally valid when the transition is smooth, and in several implementations this is advantageous numerically, as it leads to a bounded Flux operator.
} 
Subtracting the two expressions in Eqs. (21) and (23) gives the the $T$-matrix as:

$$
\begin{aligned}
& t\left(\mathbf{k}, \mathbf{k}_{i}, \hat{\mathbf{p}}, \hat{\mathbf{p}}_{i}\right) \frac{\partial k}{\partial \omega} \\
& \quad=i\left\langle\psi_{0}(\mathbf{k}, \hat{\mathbf{p}})|H \bar{\theta}-\bar{\theta} H| \psi_{\text {scatt }}\right\rangle,
\end{aligned}
$$

where $\bar{\theta}$ is defined as 1 in the interaction region, and zero outside. This leads to:

$$
\begin{aligned}
\left\langle\psi_{0}|H \bar{\theta}-\bar{\theta} H| \psi_{\text {scatt }}\right\rangle & =\left\langle\psi_{0}|H \bar{\theta}-\bar{\theta} E| \psi_{\text {scatt }}\right\rangle \\
& =\left\langle\psi_{0}|H \bar{\theta}-E \bar{\theta}| \psi_{\text {scatt }}\right\rangle \\
& =\left\langle\psi_{0}\left|H \bar{\theta}-H_{0} \bar{\theta}\right| \psi_{\text {scatt }}\right\rangle \\
& =\left\langle\psi_{0}\left|H-H_{0}\right| \psi_{\text {scatt }}\right\rangle
\end{aligned}
$$

where the last equality follows from the fact that $w-w_{0}$ is localized and from it results the usual expression for the $T$-Matrix as an integral over $H-H_{0}$ :

$$
\begin{aligned}
& t\left(\mathbf{k}, \mathbf{k}_{i}, \hat{\mathbf{p}}, \hat{\mathbf{p}}_{i}\right) \\
& \quad=i\left(\frac{d k}{d \omega}\right)^{-1}\left\langle\psi_{0}(\mathbf{k}, \hat{\mathbf{p}})|H-H| \psi_{\text {scatt }}\right\rangle,
\end{aligned}
$$

where now the surface is defined in front of $\mathbf{k}$, i.e. $s_{0}$ is now here a large positive number. Using the dispersion relation for light:

$$
\omega=k / \sqrt{\varepsilon},
$$

we obtain the final expression:

$$
\begin{aligned}
& t\left(\mathbf{k}, \mathbf{k}_{i}, \hat{\mathbf{p}}, \hat{\mathbf{p}}_{i}\right) \\
& \quad=\frac{i}{\sqrt{\varepsilon}}\left\langle\psi_{0}(\mathbf{k}, \hat{\mathbf{p}})\left|H-H_{0}\right| \psi_{\text {scatt }}\left(\mathbf{k}_{i}, \hat{\mathbf{p}}_{i}\right)\right\rangle .
\end{aligned}
$$

\subsection{Energy-dependent wavefunction from time-dependent approach}

To evaluate the $T$-matrix, which is a time-independent quantity [see Eq. (28)], we move now to a timedependent approach. This will allow the simultaneous calculation of many transition matrix elements at once. There are several ways for doing this, but here we consider iterative methods which use very efficiently the sparsity of the Hamiltonian.

We consider problems where the incoming waves are propagating along the $z$-axis and the initial function is constant in the $x-y$ direction. Thus we introduce an initial wave-packet in the $z$-direction denoted as

$$
\varphi_{\text {in }}(x, y, z, \hat{\mathbf{p}}, t=0)=\eta_{\text {in }}\left(z, \hat{\mathbf{p}}_{i}, t=0\right) N(x, y)
$$

where

$$
N(x, y)=1
$$

Remember that $\eta$ is a 6-dimensional vector with polarization $\hat{\mathbf{p}}_{i}$. As it stands, it would be hard to propagate in time the wave-packet $\left|\varphi_{\text {in }}\right\rangle$ since it would require a grid that is formally infinitely long in $x, y$; for that purpose, we use the two-grid formulation which is reviewed below. Note that we will consistently use different symbols for a wave-packet $\varphi$ and for planewaves or scattering states $\psi$. We can view $\varphi_{\text {in }}$ as a linear combination of time-independent wavefunctions, each associated with an incoming planar wave emerging from the left along the $z$-axis and uniform along the $x-y$ coordinates:

$$
\left|\varphi_{\mathrm{in}}(t=0)\right\rangle=\int a_{\omega}\left|\psi_{\mathrm{in}}(\omega)\right\rangle d \omega
$$

where $a_{\omega}$ is a coefficient showing how much of the initial wave-function $\psi_{\text {in }}(\omega)$ of energy $\omega$ is in the initial wave-packet, as explained below. Because $\psi_{\text {in }}$ is a plane wave, we can extract $a_{\omega}$ directly out of $\varphi_{\text {in }}$ using a 1-dimensional Fourier transform (taken over $z$ for $x=y=0)$ :

$$
a_{\omega}=\sqrt{\varepsilon_{b} \psi} f_{\hat{\mathbf{p}}}^{\dagger} \int \exp (-i k(\omega) z)^{\dagger} \varphi_{\mathrm{in}}(z) d z .
$$

This relation is based on the following plane waves orthogonally relation, including the dispersion relation Eq. (27):

$$
\begin{aligned}
& \int \psi_{0}\left(\mathbf{r} ; k(\omega) \hat{\mathbf{z}}, \hat{\mathbf{p}}_{i}\right)^{\dagger} \psi_{0}\left(\mathbf{r} ; k\left(\omega^{\prime} \hat{)} \mathbf{z}, \hat{\mathbf{p}}_{i}\right) d z\right. \\
& =\frac{1}{4 \pi^{2}} \delta\left(k(\omega)-k\left(\omega^{\prime}\right)\right)=\frac{\delta\left(\omega-\omega^{\prime}\right)}{4 \pi^{2} \sqrt{\varepsilon_{b}}} .
\end{aligned}
$$

The choice of $\varphi_{\text {in }}$ is such that the wave packet is spatially localized in the incoming asymptote $(z \rightarrow-\infty)$. This means that the wave packet can be equally well written as:

$$
\left|\varphi_{\text {in }}(t=0)\right\rangle=\int a_{\omega}\left|\psi_{\text {scatt }}(\omega)\right\rangle d \omega
$$


Now let the clock tick and propagate $\varphi_{\text {in }}$ in time, using the "time-dependent Schrödinger equation $i \dot{\varphi}=H \varphi$ " with solution:

$$
\varphi(t)=e^{-i H t} \varphi_{\mathrm{in}}
$$

The scattering state at energy $\omega$ can be projected out of $\varphi_{\text {in }}$ using:

$$
\begin{aligned}
\left|\psi_{\text {scatt }}(\omega)\right\rangle= & \frac{1}{a_{\omega}} \delta(\omega-H)\left|\varphi_{\text {in }}\right\rangle \\
= & \frac{1}{2 \pi a_{\omega}}\left[\int_{0}^{\infty} \exp (i(\omega-H) t) d t\right. \\
& \left.+\int_{-\infty}^{0} \exp (i(\omega-H) t) d t\right]\left|\varphi_{\text {in }}\right\rangle \\
= & \frac{i}{2 \pi a_{\omega}}\left[G(\omega)\left|\varphi_{\text {in }}\right\rangle+|\zeta(\omega)\rangle\right],
\end{aligned}
$$

where the Green's function is defined as: $G(\omega)=$ $\frac{1}{\omega-H} . \zeta(\omega)$ is the part associated with the initial motion of the wave-packet (from -infinity to zero) and can be ignored. Scattering calculations usually do not calculate $G(\omega)$ but modify the integral to include an absorbing part; this amounts to rewriting

$$
G(\omega)=\frac{1}{\omega-\tilde{H}},
$$

where $\tilde{H}=H-i \Gamma$ includes an absorbing potential assuring convergence of the integral in Eq. (36). In scattering calculations over a large grid, $\Gamma$ is an imaginary potential padding the grid. But here, the calculations are more complicated, since the initial wavefunction is infinitely extended in the $x, y$ directions. This difficulty is resolved by the two-grid formalism, as explained in the next section.

Using Eqs. (28), (32) and (36) a compact expression for the $T$-matrix is then:

$$
\begin{aligned}
t\left(\mathbf{k}, \mathbf{k}_{i}, \hat{\mathbf{p}}, \mathbf{p}_{i}\right) & \\
= & \frac{i\left\langle\psi_{0}(\mathbf{k}, \hat{\mathbf{p}})\left|H-H_{0}\right| \int \varphi(\mathbf{r}, t) \exp (i \omega t) d t\right\rangle}{2(\pi)^{3 / 2} \sqrt{\varepsilon_{b}} \int \exp (-i k z) f_{\hat{\mathbf{p}}}^{\dagger} \varphi_{\mathrm{in}}(z) d z} .
\end{aligned}
$$

This expression can be used with the wave-packet $\varphi(\mathbf{r}, t)$ represented on a grid and filtered for each energy, and then the overlapped with $\psi_{0}(\mathbf{k}, \hat{\mathbf{p}})$; or alternately, one can first calculate the overlap with each $\psi_{0}(\mathbf{k}, \hat{\mathbf{p}})$ at each time-step and only then filter this overlap. ${ }^{31}$ The choice between the methods depends on the application, as explained in the appendix.

\section{The Two-Grid Formalism}

To alleviate the problem due to the fact that the initial wavefunction is defined on an extended grid, we write the time-dependent wave-packet as:

$$
\varphi=\chi+\phi .
$$

where the $i$ sub-script is omitted. There is a freedom in the choice of $\phi$ and $\chi$. For this application, it is appropriate to use a version in which $\phi$ is determined by a zeroth-order Hamiltonian, in which the index of refraction is independent of $x$ and $y$, and is either a constant or dependent purely on $z$, the propagation direction. (Such a version is different from the projection operator formalism used in chemical applications.) Specifically, we write:

$$
i \frac{d \phi}{d t}=H_{0} \phi, \quad \phi(t=0)=\varphi_{\mathrm{in}},
$$

where $H_{0}$ is analogous to $H$ but with $\varepsilon$ replaced by $\varepsilon_{b}(z)$, as in Eq. (6). Since the index of refraction and the initial wavepacket are both independent of $x, y$, it follows that they would always be independent of $x, y$, so that

$$
\phi(x, y, z, \hat{\mathbf{p}}, t)=\eta(z, \hat{\mathbf{p}}, t) .
$$

The propagation of $\phi$ is therefore completely equivalent to the evolution of $\eta$ under the $z$-dependent Hamiltonian

$$
i \frac{d \eta}{d t}=h_{0} \eta
$$

where

$$
h_{0}=i\left(\begin{array}{ccccc} 
& & 0 & \frac{-1}{\sqrt{\varepsilon_{b}}} \frac{\partial}{\partial z} & 0 \\
& 0 & \frac{1}{\sqrt{\varepsilon_{b}}} \frac{\partial}{\partial z} & 0 & \\
0 & 0 & 0 & & \\
0 & \frac{1}{\sqrt{\varepsilon_{b}}} \frac{\partial}{\partial z} & 0 & & \\
\frac{-1}{\sqrt{\varepsilon_{b}}} \frac{\partial}{\partial z} & 0 & & &
\end{array}\right) .
$$


The equation for $\chi$ is now:

$$
i \frac{d \chi}{d t}=\left(H-H_{0}\right) N \eta+H \chi
$$

Note that $\eta$ is a function of $z$ so the grid on which $\eta$ is represented is a 1-dimensional grid, while $\chi$ is a 3-dimensional wave packet, represented on a 3dimensional grid. As discussed in the previous section, we actually need to modify Eqs. (40) and (44) by adding absorbing potentials, so that the waves reaching the 1-dimensional and 3-dimensional grid boundaries are not reflected. We denote these absorbing potentials as $\Gamma_{\phi}$ and $\Gamma_{\chi}$ :

$$
\begin{gathered}
i \frac{d \eta}{d t}=\left(h_{0}-i \Gamma_{\phi}\right) \eta, \\
i \frac{d \chi}{d t}=\left(H-H_{0}\right) N \eta+\left(H-i \Gamma_{\chi}\right) \chi, \\
\chi(t=0)=0 .
\end{gathered}
$$

The introduction of $\Gamma_{\chi}$ is essential to eliminate the need to represent $\chi$ beyond the interaction area. The final equations can be recast in the following homogeneous Hamiltonian form:

$$
\begin{aligned}
i \frac{d}{d t}\left(\begin{array}{l}
\chi \\
\eta
\end{array}\right) & =\mathbf{H}-i \boldsymbol{\Gamma}\left(\begin{array}{l}
\chi \\
\eta
\end{array}\right) \\
& \equiv\left(\begin{array}{cc}
H-i \Gamma & \left(H-H_{0}\right) N \\
0 & h_{0}-i \Gamma_{\phi}
\end{array}\right)\left(\begin{array}{l}
\chi \\
\eta
\end{array}\right) .
\end{aligned}
$$

This form allows for a very convenient and efficient propagation, in particular the Chebyshev propagator $^{19}$ (which works well despite the nonHermitian nature of $\mathbf{H}^{26}$ ). The filter of this combined two grids wavefunction yields the Green's function for H:

$$
\begin{aligned}
\left(\begin{array}{l}
\chi(\omega) \\
\phi(\omega)
\end{array}\right) & =\frac{1}{2 \pi a_{\omega}} \int_{0}^{\infty} \exp (i \omega t)\left(\begin{array}{c}
\chi(t) \\
\phi(t)
\end{array}\right) d t \\
& =\frac{1}{2 \pi i a_{\omega}} \frac{1}{(\omega-\mathbf{H}+i)}\left(\begin{array}{c}
0 \\
\varphi_{\text {in }}
\end{array}\right) \\
& \equiv \frac{1}{2 \pi i a_{\omega}} G_{\mathbf{H}}(\omega)\left(\begin{array}{c}
0 \\
\varphi_{\text {in }}
\end{array}\right) .
\end{aligned}
$$

The advantage of this form is that only one Green's function is needed and will be used to extract many
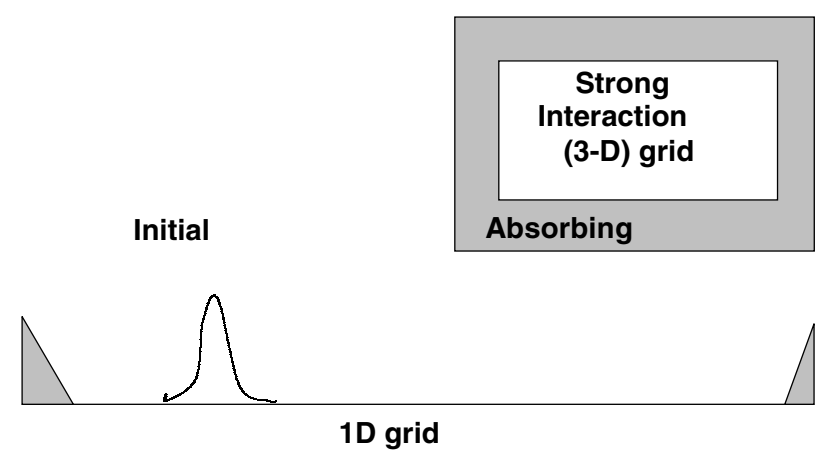

Fig. 1. Schematics of the 2 grid approach. Shown, a small 3-D grid, and a simple grid showing the strong-interaction region. The shaded regions are covered with an absorbing potential. A localized initial wave-packet is also shown.

energies; the extra price, in terms of the two-grid propagation, is negligible, since the $3 \mathrm{D}$ grid is small (covering just the strong interaction region); the other grid, while longer (to cover the initial function region) is just 1D. (See Fig. 1.)

The initial wave-packet used here is an $x$-polarized wave-packet

$$
\varphi_{\text {in }}(z, t=0)=\sqrt{\frac{1}{\pi \sigma}} \exp \left(-\left(z-z_{0}\right)^{2} / 2 \sigma^{2}\right)\left(\begin{array}{l}
1 \\
0 \\
0 \\
0 \\
0 \\
0
\end{array}\right) .
$$

This initial wave-packet is real, and therefore the whole simulation is real (the wave-packet is purely real, without an initial magnetic field) and the only necessary complex algebra enters into the calculation of $\psi(\omega)$. The fact that the initial wavepacket contains both incoming and outgoing components (positive and negative $k_{z}$ and polarization components) is because the outgoing components are absorbed by the left absorbing potential (on the 1-D grid) and they do not influence the results.

\section{Summary of Approach}

The resulting approach is summarized as follows:

(1) Place an initial wavefunction, $\left(\begin{array}{c}0 \\ \varphi_{\text {in }}\end{array}\right)$ far from the interaction region. 
Table 1. Some simulation parameters.

\begin{tabular}{|l|l|}
\hline $1 \mathrm{D}$ grid extent $($ for $\phi)$ & {$[-60,15]$} \\
\hline Strong-interaction grid extent (for $\chi$ ) in each dimension & {$[-15,15]$} \\
\hline Time-step & 1 \\
\hline Total time & 120 \\
\hline Chebyshev min/max parameters & $-5,5$ \\
\hline Absorbing potential width & $10($ or $6-$ see below) \\
\hline Absorbing potential height & 2 \\
\hline Index of refraction parameters & $\varepsilon_{0}=1 \quad \beta=0.5 \quad b=5$. \\
\hline
\end{tabular}

(2) Propagate this wavefunction in time,

$$
\left(\begin{array}{c}
\chi(t) \\
\varphi(t)
\end{array}\right)=\exp (-i \mathbf{H}-i \boldsymbol{\Gamma}) t\left(\begin{array}{c}
0 \\
\varphi_{\text {in }}
\end{array}\right),
$$

using any of the established approaches (especially, Chebyshev ${ }^{19}$ or Chebyshev with modified coefficients to account for the absorbing potentials ${ }^{22}$ ).

(3) Filter this wavefunction at several energies (i.e., frequencies), to $\operatorname{obtain}\left(\begin{array}{ll}\chi & (\omega) \\ \varphi & (\omega)\end{array}\right)$; again, either a direct filtering of a time-dependent wavefunction or a filtering which takes into account the properties of the Chebyshev propagators. ${ }^{22,32,33}$

(4) Determine the transition amplitudes at any desired direction and polarization from Eq. (38).

Several variants, completely equivalent to those in scattering theory, are presented in the appendix.

\section{Model Simulation}

We simulated the Maxwell equation for a very simple polarization function

$$
\varepsilon(\mathbf{r})=\left\{\begin{array}{cc}
\varepsilon_{0} & r>b \\
\varepsilon_{0}\left(1-\beta \cos ^{2}(\pi r / 2 b)\right) & r \leq b
\end{array}\right.
$$

where parameters are presented in Table $1 . \quad$ We used dimensionless units; for simplicity, it can be assumed that lengths are in micron and times are in $(\mathrm{c} / \text { micron })^{-1}=3.3 \cdot 10^{-15} \mathrm{sec}$.

Some simulation parameters are presented in Table 1. The absorbing potential was parabolic and

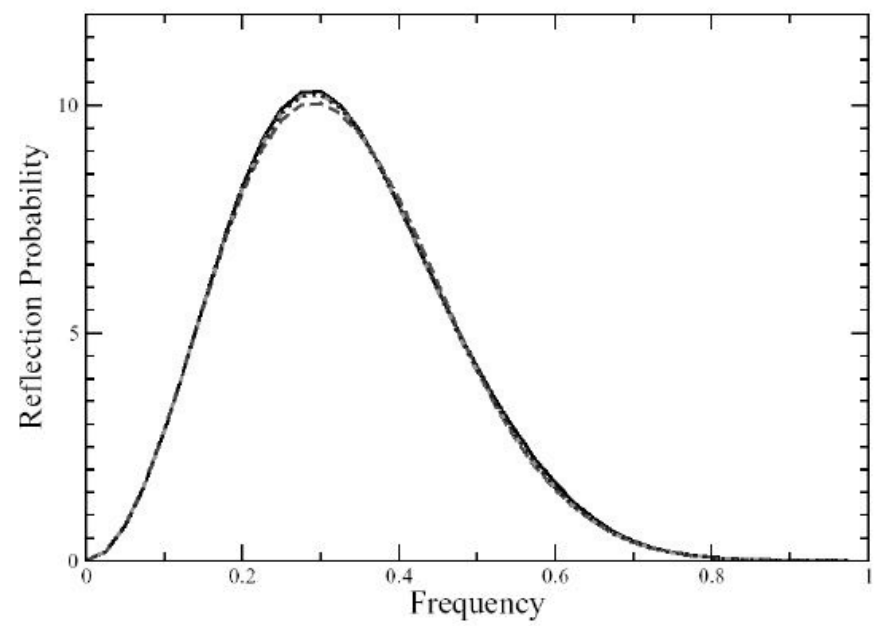

Fig. 2. The squared transmission probability density, defined here as $(2 \pi)^{3}\left|t\left(\mathbf{k}, \mathbf{k}_{i}, \hat{\mathbf{p}}, \hat{\mathbf{p}}_{i}\right)\right|^{2}$, for a reflected wave, for several different grid spacing and absorbing potentials, as explained in the text; the only curve which shows (slight) deviation from the rest is the dashed-curve, where the absorbing potential width is set at 6 rather than 10 . The other curves, associated with different grids and grid spacings, are all virtually identical.

extended over all 6 sides of the 3-D grid and the two sides of the 1-D grid. The Chebyshev $\min / \max$ parameters are smaller than those nominally needed (the $\min / \max$ eigenvalues of the $\mathbf{H}$ operator) but were sufficient since $d t$ was small. Our goal was to verify insensitivity to grid parameters. This is shown in Fig. 2, which superimposes the squared modulus of the transition amplitudes for several cases, as follows:

40 points in each $3 \mathrm{D}$ direction;

30 points in each $3 \mathrm{D}$ direction;

20 points in each $3 \mathrm{D}$ direction; and 
30 points in each $3 \mathrm{D}$ direction with an absorbing potential width of 6 .

We have also done a very large-scale simulation with $100 \times 100 \times 100$ points (with a $3 \mathrm{D}$ grid extent of $[-35,35]$ in each dimension) where we filtered the wave-packet only at a few energies. The goal of this case was simply to show that a simulation over very large grids is viable.

The most encouraging aspect of the simulation was their good scaling with problem size. Even without code optimization (for example, we did not take into account that the simulation can use only real algebra - this would be done in an upcoming publication), a $40 \times 40 \times 40$ points simulation with 100 frequencies simulation took 8 hours on a $1 \mathrm{GHz}$ workstation (about 10 times more than for $20 \times 20 \times 20$ ), and the $100 \times 100 \times 100$ simulations took about 20 times longer - since the simulation is essentially linear in overall grid size. When we sample at many frequencies, about half the CPU time was occupied with filtering the wave-packet at the different frequencies, and this is also where most of the memory was used.

\section{Discussion}

This work shows that Maxwell's equations can be very easily and efficiently solved with the two-grid formalism. The equations are homogenous and the results are extracted for a large set of photon energies from a single calculation. Because two grids are used, the initial wave-packet can be quite compact without any approximation. In addition, several alternative approaches are discussed in the appendix.

There are several directions for extending this work, both methodologically and for applications:

Extend the two-grid formalism to waves that propagate in a periodic crystal (so $H_{0}$ is associated with a periodic crystal, while $H$ includes a local departure from $H_{0}$ ), both for waves that propagate along one of the crystal axis and waves that emanate from an arbitrary direction. This is feasible, as would be shown in an upcoming publication.

Extend the formalism further to cases with time-dependent perturbations, e.g. due to excited molecules. This is feasible, for example, with the $t, t^{\prime}$ formalism. ${ }^{34,35}$

The one down side in the simulation is that for every energy value (frequency), a different resolved grid function is extracted. This adds to the storage, and also to the CPU time (although this is not significant unless more than about 50 frequencies are used, since most of the effort is in applying the Hamiltonian operator). The way to avoid the additional storage, as explained above, is that instead of first filtering the wavefunction and then overlapping it with a final state, one reverses the order (first overlap the time-dependent wavefunction with the final function and then filter). This results in storage saving at the expense of extra CPU, since the overlap with each final state needs to be calculated at each time-step. If the wavefunction is transformed to a different coordinate system, associated with the wave-vector of the final state, then the extra CPU time required is minimized; however, this removes one of the advantages of the wave-packet techniques: that a host of final amplitudes (each associated with a different wavevector direction as well as energy) is extracted from a single wave packet.

Other alternatives from scattering that can be employed are the product-reactant decoupling approach. ${ }^{36}$

Even though the improvements are desirable, the formalism, even as written, is directly applicable to large-scale photonic band-gap studies of deviation from periodicity, as will be described in upcoming simulations.

\section{Acknowledgment}

We gratefully acknowledge funding from the NSF, BSF, PRF and the Israel Science Foundation. The authors thank discussions with Vicki Colvin.

\section{Appendix}

In the appendix, we show various extensions and other options feasible. The extensions have either been reported earlier or are slight variations of previous work, and are presented only for completeness.

First, the propagation equation can be written directly for $(\psi, \eta)$ rather than $(\chi, \eta)$ yielding:

$$
\begin{aligned}
\left(\begin{array}{c}
\psi(E) \\
\eta(E)
\end{array}\right) & =\frac{1}{i\left(E-\mathbf{H}^{\prime}+i \boldsymbol{\Gamma}\right)}\left(\begin{array}{c}
0 \\
\eta_{i}
\end{array}\right) \\
& \equiv G_{\mathbf{H}^{\prime}}(\omega)\left(\begin{array}{c}
0 \\
\eta_{i}
\end{array}\right)
\end{aligned}
$$


where

$$
\mathbf{H}^{\prime}-i \boldsymbol{\Gamma}=\left(\begin{array}{cc}
H-i \Gamma & i \Gamma \\
0 & h_{0}-i \Gamma_{\phi}
\end{array}\right),
$$

associated with a time-dependent propagation of the form:

$$
i \frac{d}{d t}\left(\begin{array}{l}
\psi \\
\phi
\end{array}\right)=\left(\begin{array}{cc}
H-i \Gamma & i \Gamma \\
0 & h_{0}-i \Gamma_{\phi}
\end{array}\right)\left(\begin{array}{l}
\psi \\
\phi
\end{array}\right) .
$$

Another alternative is to write the equations directly using $G(\omega)$, i.e. without using the two-grid formalism.

$$
\psi(\omega)=\phi_{i}(\omega)+\frac{1}{\omega-H+i \Gamma}\left(H-H_{0}\right) \phi_{i}(\omega),
$$

or equivalently:

$$
\psi(\omega)=\frac{i}{\omega-H+i \Gamma} \Gamma \phi_{i}(\omega) .
$$

This algorithm is simpler conceptually, since it involves the inversion of the Hamiltonian matrix of a single 3D grid over a small region; however, it requires a different inversion for each energy so that one loses a major advantage of wave-packet techniques the ability to extract results for many energies at once. It is appropriate when preconditioning is used to reduce the number of Hamiltonian evaluations. ${ }^{35}$

\section{References}

1. R.F. Service, Science 295, 2399 (2002).

2. E. Yablonovitch, T.J. Gmitter and K.M. Leung, Phys. Rev. Lett. 67, 2295 (1991).

3. H. Pier, E. Kapon and M. Moser, Nature 407, 880 (2000).

4. E. Yablonovitch, Science 289, 557 (2000).

5. J. Pendry, Science 285, 1687 (1999).

6. R. Rengarajan, P. Jiang, D.C. Larrabee, V. L. Colvin and D.M. Mittleman, Phys. Rev. B64, 205103 (2001).

7. K.M. Leung and Y.F. Liu, Phys. Rev. Lett. 65, 2646 (1990).

8. R. Kemp and J.E. Inglesfield, Phys. Rev. B65, 115103 (2002).

9. J.B. Pendry and A. Mackinnon, Phys. Rev. Lett. 69, 2772 (1992).
10. D. Felbacq, B. Guizal and F. Zolla, J. Opt. a-Pure Appl. Opt. 2, L30 (2000).

11. B. Gralak, S. Enoch and G. Tayeb, J. Opt. Soc. Am. Opt. Image Sci. Vision 17, 1012 (2000).

12. T.T. Prasad and C.V. Mittleman, Phys. Rev. B67, 165103 (2003).

13. D. Neuhauser, R.S. Judson, M. Baer and D.J. Kouri, J. Chem. Soc. Faraday Trans. 93, 727 (1997).

14. D. Neuhauser, J. Chem. Phys. 100, 9272 (1994).

15. O.A. Sharafeddin, R.S. Judson, D.J. Kouri and D.K. Hoffman, J. Chem. Phys. 93, 5580 (1990).

16. D.H. Zhang and J.Z.H. Zhang, J. Chem. Phys. 100, 2697 (1994).

17. U. Manthe, T. Seideman and W.H. Miller, J. Chem. Phys. 99, 10078 (1993).

18. R.C. Mowrey, Y. Sun and D.J. Kouri, J. Chem. Phys. 91, 6519 (1989).

19. R. Kosloff, J. Phys. Chem. 92, 2087 (1988).

20. D. Neuhauser, M. Baer, R.S. Judson and D.J. Kouri, Comput. Phys. Commun. 63, 460 (1991).

21. G. Katz, R. Baer and R. Kosloff, Chem. Phys. Lett. 239, 230 (1995).

22. V.A. Mandelshtam and H.S. Taylor, J. Chem. Phys. 103, 2903 (1995).

23. D. Neuhauser and M. Baer, J. Chem. Phys. 91, 4651 (1989).

24. D. Neuhasuer and M. Baer, J. Chem. Phys. 90, 4351 (1989).

25. D. Neuhauser and M. Baer, J. Chem. Phys. 92, 3419 (1990).

26. D. Neuhauser, Chem. Phys. Lett. 200, 173 (1992).

27. J.P. Mattia, E.R. Brown and C.D. Parker, Phys. Rev. B57, 1308 (1998).

28. D.J. Kouri and D.K. Hoffman, unpublished.

29. T. Seideman and W.H. Miller, J. Chem. Phys. 97, 2499 (1992).

30. R.S. Judson, D.B. McGarrah, O.A. Sharafeddin, D.J. Kouri and D.K. Hoffman, J. Chem. Phys. 94, 3577 (1991).

31. D. Neuhauser, J. Chem. Phys. 93, 2611 (1990).

32. R. Baer, Y. Zeiri and R. Kosloff, Phys. Rev. BCondens. Matter 54, R5287 (1996).

33. U. Peskin, R. Kosloff and N. Moiseyev, J. Chem. Phys. 100, 8849 (1994).

34. U. Peskin, W.H. Miller and A. Edlund, J. Chem. Phys. 103, 10030 (1995).

35. J.Q. Dai and J.Z.H. Zhang, J. Chem. Soc. Faraday Trans. 93, 699 (1997). 
Copyright of Journal of Theoretical \& Computational Chemistry is the property of World Scientific Publishing Company and its content may not be copied or emailed to multiple sites or posted to a listserv without the copyright holder's express written permission. However, users may print, download, or email articles for individual use. 
Copyright of Journal of Theoretical \& Computational Chemistry is the property of World Scientific Publishing Company and its content may not be copied or emailed to multiple sites or posted to a listserv without the copyright holder's express written permission. However, users may print, download, or email articles for individual use. 